\title{
Biomechanics of a flexible sublaminar connector in long-segment thoracic fixation
}

\author{
Nestor G. Rodriguez-Martinez, MD, ${ }^{1}$ Sam Safavi-Abbasi, MD, ${ }^{1}$ Luis Perez-Orribo, MD,, \\ Anna G. U. S. Newcomb, MS, ${ }^{1}$ Phillip M. Reyes, BSE,, Galyna Loughran, PhD, ${ }^{2}$ \\ Nicholas Theodore, MD, ${ }^{1}$ and Neil R. Crawford, PhD'
}

${ }^{1}$ Spinal Biomechanics, Division of Neurological Surgery, Barrow Neurological Institute, St. Joseph's Hospital and Medical Center, Phoenix, Arizona; and '2Zimmer Spine, Minneapolis, Minnesota

OBJECTIVE The Universal Clamp Spinal Fixation System (UC) is a novel sublaminar connection for the spine that is currently used in conjunction with pedicle screws at the thoracic levels for the correction of scoliosis. This device allows the surgeon to attach rods and incorporate a pedicle screw construction. The flexible composition of the UC should provide flexibility intermediate to the uninstrumented spine and an all-screw construct. This hypothesis was tested in vitro using nondestructive flexibility testing of human cadaveric spine segments.

METHODS Six unembalmed human cadaveric thoracic spine segments from T-3 to T-11 were used. The specimens were tested under the following conditions: 1) intact; 2) after bilateral screws were placed at T4-T10 and interconnected with longitudinal rods; 3 ) after placement of a hybrid construction with screws at T-4, T-7, and T-10 with an interconnecting rod on one side and screws at T- 4 and T-10 with the UC at T5-9 on the contralateral side; (4) after bilateral screws were placed at T-4 and T-10 and interconnected with rods and bilateral UC were placed at T5-9; and 5) after bilateral screws at T-4 and T-10 were placed and interconnected with rods and bilateral sublaminar cables were placed at T5-9. Pure moments of $6.0 \mathrm{Nm}$ were applied while optoelectronically recording 3D angular motion.

RESULTS Bilateral UC placement and bilateral sublaminar cables both resulted in a significantly greater range of motion than bilateral pedicle screws during lateral bending and axial rotation, but not during flexion or extension. There were no differences in stability between bilateral UC and bilateral cables. The construct with limited screws on one side and UC contralaterally showed comparable stability to bilateral UC and bilateral cables.

CONCLUSIONS These results support using the UC as a therapeutic option for spinal stabilization because it allows comparable stability to the sublaminar cables and provides flexibility intermediate to that of the uninstrumented spine and an all-screw construct. Equivalent stability of the hybrid, bilateral UC, and bilateral cable constructs indicates that 6 -level UC provides stability comparable to that of a limited (3-point) pedicle screw-rod construct.

http://thejns.org/doi/abs/10.3171/2015.4.SPINE14999

KEY WORDS biomechanics; pedicle screw; sublaminar wiring; thoracolumbar; thoracic

$\mathrm{C}$ ORRECTION of scoliosis has been performed using various fixation systems. All-screw constructs are currently the most popular. ${ }^{11}$ Eduardo Luque introduced the concept of segmental posterior spine instrumentation with sublaminar cables in $1976 .{ }^{15}$ Modifications to this technique have been made, ${ }^{22}$ but sublaminar implants are still in use. Recent studies demonstrate that soft sublaminar implants distribute stress over a larger area of the lamina, reducing the risk of laminar fracture while providing equivalent reduction. ${ }^{1,10}$
The Universal Clamp Spinal Fixation System (UC) (Zimmer Spine) is a novel sublaminar implant currently used in conjunction with pedicle screws at the thoracic levels to correct scoliosis. The UC is a flexible polyester sublaminar loop that attaches to the pedicle screw interconnecting rod, allowing greater flexibility than a screw or metallic sublaminar cable. Using the UC as an option for correcting adolescent idiopathic scoliosis has been described before with good clinical results. ${ }^{12,15,18}$ A hybrid construction (UC together with rigid screw fixation) has 
been shown to be an effective therapeutic option for treating scoliosis. ${ }^{13,16,19}$ The biomechanical properties of UC have been studied in short constructs. ${ }^{9}$ However, the device has not been evaluated in long posterior constructs. ${ }^{9}$

We hypothesized that UC would provide flexibility intermediate to that of the intact spine and spine instrumented with an all-screw construct. Because of its nonrigid nature, we also hypothesized that the UC would allow a smaller zone of laxity but a larger range of motion (ROM) than sublaminar cables or all-screw constructs. In this study, these hypotheses were tested in vitro using nondestructive flexibility testing of human cadaveric spine segments.

\section{Methods}

Six unembalmed human cadaveric thoracic spine segments from T3-11 were used (4 male and 2 female; mean age 46 years [range 29-59 years]). The specimens were carefully cleaned of muscle tissue while all ligaments, joint capsules, and discs were kept intact. Dual-energy x-ray absorptiometry scans were performed on the L-4 vertebra of each specimen to assess bone mineral density before dissection of the T3-11 segment. None of the specimens were osteoporotic. Plain film radiographs were taken, and the history was reviewed to ensure that no specimens had obvious flaws, metastatic disease, osteophytes, disc narrowing, or joint arthrosis. Specimens were prepared for biomechanical testing by reinforcing the T-11 segment with household wood screws, embedding the segment in a block of fast-curing resin (Smooth-Cast 300Q, SmoothOn, Inc.), and attaching the segment to the base of the testing apparatus. The T-3 vertebra was also reinforced with household screws and embedded in resin in a cylindrical metal fixture for load application.

The biomechanical characteristics of the 6 specimens were determined under 5 conditions: 1) intact; 2) after bilateral screws were placed at T4-10 and interconnected with longitudinal rods; 3) after a hybrid construction was placed with screws at T-4, T-7, and T-10 with an interconnecting rod on one side and screws placed at T- 4 and T-10 with the UC placed at T5-9 on the contralateral side; 4) after bilateral screws were placed at T4 and T10 and interconnected with rods and bilateral UC at intervening levels; and 5) after bilateral screws were placed at T4 and T10 and interconnected with rods and bilateral sublaminar cables at the intervening levels (Fig. 1).

Placement of the pedicle screws was done using the "freehand" technique. The screws were oriented parallel to the superior endplate of each vertebra. After localization of the entry point, an acorn-tipped, 3-mm bur was used to create a posterior cortical breach approximately $5 \mathrm{~mm}$ deep. After the cortical bone was drilled through, a thoracic "gearshift" probe was placed in the base of the pedicle. The pedicle tract was tapped using a 3-mm tap. Pedicle screws that measured $4.5 \times 35 \mathrm{~mm}$ were inserted bicortically. After the rod was removed in the second condition, $5.5 \times 40-\mathrm{mm}$ rescue screws were placed if it was noted that the screws were loose. Radiographs were taken to verify the correct placement of the screws.

Under the third condition, the UC was placed according to the manufacturer's instructions. The UC band was sublaminarly passed caudal to rostral, starting near the transverse process. After the UC bands were attached to the vertebrae, the rod was attached with the clamp in a medial orientation and assembled with a locking screw with a tapered screw starter. The band was tightened by using the reduction tool and by applying $700 \mathrm{~N}$ of tension following the UC surgical technique.

Under all conditions for each specimen, an apparatus was used in which a system of cables and pulleys imparted nondestructive, nonconstraining torque in conjunction with a standard servohydraulic test system. ${ }^{2}$ With pure moment loading, the same load is distributed to each level of the spine, ensuring an equivalent comparison among all levels and among specimens regardless of the distance from the loading point. ${ }^{17}$ Maximum loads of $6.0 \mathrm{Nm}$ were applied about the appropriate anatomical axes to induce bending or twisting in each plane of motion: flexion and extension, left and right lateral bending, and left and right axial rotation. In each loading direction, 3 preconditioning cycles were applied at $6.0 \mathrm{Nm}$ for 60 seconds each, after
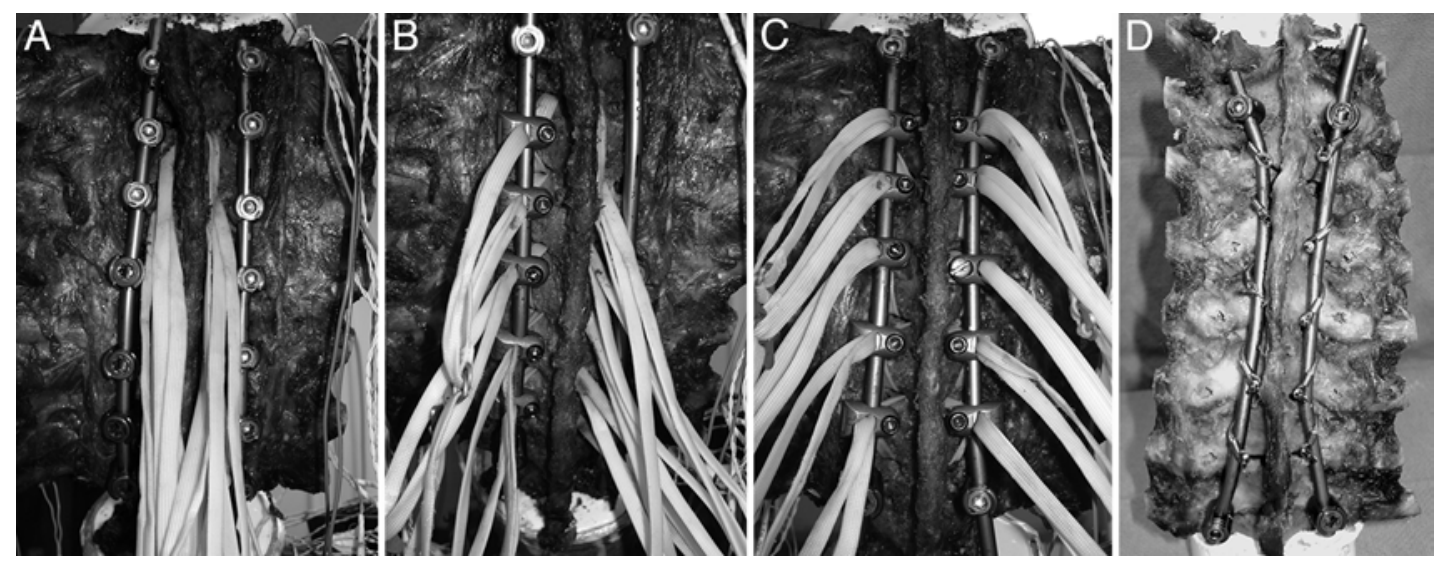

FIG. 1. Posterior photographs of representative specimens under the studied fixation conditions (the intact condition is not shown). A: Bilateral pedicle screws and interconnecting rods at all levels. The UC straps are sublaminarly passed but not connected. B: Pedicle screws at T-4, T-7, and T-10 on the right, plus unilateral UC at T5-9 and pedicle screws at T-4 and T-10 and on the left. C: Bilateral UC at T5-9 with bilateral pedicle screws at T-4 and T-10. D: Bilateral sublaminar cables at T5-9 with bilateral pedicle screws at T-4 and T-10. 
hich the specimen was allowed to rest without a load for 60 seconds. During the data collection cycle, the load was applied quasistatically in $1.5-\mathrm{Nm}$ increments, with each incremental load held for 45 seconds to a maximum of $6.0 \mathrm{Nm}$. Three-dimensional specimen motion in response to the applied loads during the flexibility and compression tests was determined using the Optotrak 3020 system (Northern Digital). This system stereophotogrammetrically measures the 3D displacement of infrared-emitting markers that are rigidly attached in a noncollinear arrangement to each vertebral body on the ends of surgical guidewires that are $4 \mathrm{~cm}$ long. Three optical markers per vertebra were mounted at the T4-10 levels to independently track the motion of the segments at T4-5, T5-6, T6-7, T7-8, T8-9, and T9-10.

Custom software converted the marker coordinates to angles about each of the anatomical axes in terms of the motion segment's own coordinate system. ${ }^{3}$ The spinal angles were calculated using a technique that provides appropriate results for describing 3D spinal motion. ${ }^{5}$

From the raw load and displacement data, we quantified the mean angular ROM, angular lax zone (LZ [i.e., the portion of ROM in which the ligaments/hardware are lax]), and angular stiff zone (SZ [i.e., the portion of the ROM in which the ligaments/hardware are under tension]). ${ }^{4}$ These parameters are indicators of stability, with smaller values indicating greater stability. Stiffness is inversely proportional to SZ, with a small SZ indicating high stiffness.

Before the statistical analysis, the data were normalized by dividing the motion parameters (ROM, LZ, and SZ) under the instrumented condition by the value of that parameter for the intact condition. Normalization helps mitigate the effects of variability among specimens. The mean values of the normalized parameters from the 3 instrumented conditions were compared using 1-way repeated measure analysis of variance, followed by the HolmŠ́dák test. Probability values of $\mathrm{p}<0.05$ were considered significant.

\section{Results}

The mean ROM using hybrid fixation or bilateral UC were significantly less than that for the intact condition in all directions of loading ( $p<0.001$; Fig. 2), with the greatest stabilizing effect noted during flexion-extension ( $24 \%$ of intact with hybrid vs $23 \%$ of intact with bilateral UC) followed by lateral bending ( $40 \%$ of intact with hybrid vs $42 \%$ of intact with bilateral UC), and the least stabilizing effect was noted during axial rotation $(71 \%$ of intact with hybrid vs $68 \%$ of intact with bilateral UC). The mean ROM was significantly smaller with bilateral pedicle screws than with hybrid instrumentation in all directions of loading $(p<0.03)$, except extension $(p=0.056$; Fig. 2). The ROM under the hybrid fixation condition was not significantly different from the ROM with bilaterally placed UC during any direction of loading $(p>0.05)$. The bilaterally placed UC condition resulted in significantly greater ROM than that of the bilateral pedicle screw condition during left and right lateral bending $(\mathrm{p}<0.006)$ and left and right axial rotation $(\mathrm{p}<0.006)$, but the difference

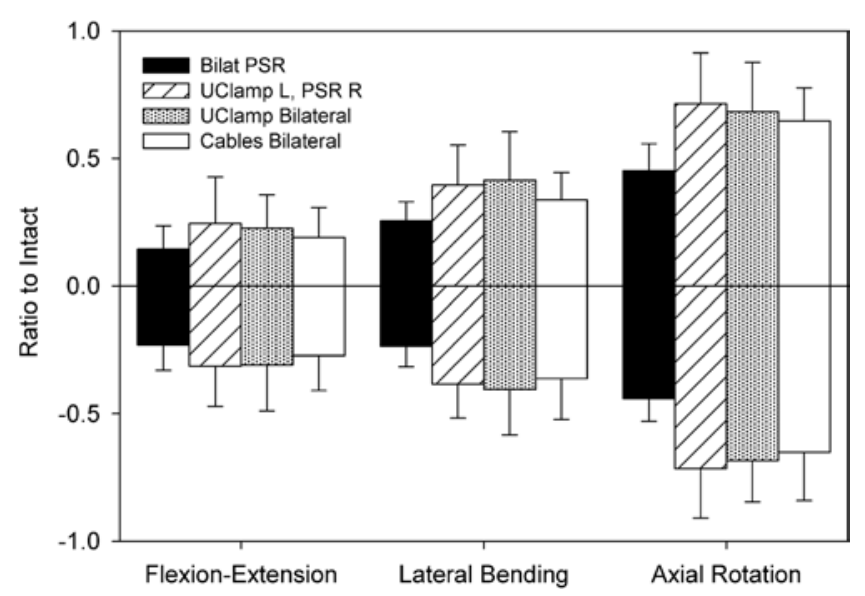

FIG. 2. Mean normalized unidirectional angular ROM across T4-10. Flexion, right lateral bending, and right axial rotation are represented as positive; extension, left lateral bending, and left axial rotation are represented as negative. The whiskers show the standard deviation. PSR = pedicle screws and rods; UClamp = Universal Clamp Spinal Fixation System.

between the 2 conditions was not significant during flexion $(p=0.065)$ or extension $(p=0.056)$. The ROM with bilateral cables was significantly greater than with bilateral pedicle screws during right lateral bending $(\mathrm{p}=0.022)$ and right and left axial rotation ( $p<0.02)$, but the difference between these 2 states was not significant during flexion $(p=0.358)$, extension ( $p=0.056)$, or left lateral bending ( $p$ $=0.191)$. ROM was not significantly different with bilateral cables than hybrid fixation $(p>0.05)$ or bilateral UC $(p>0.05)$ during any direction of loading.

The trends for the LZ (Fig. 3) were similar to those for ROM, with bilateral pedicle screws resulting in a significantly smaller LZ than hybrid fixation during axial rotation $(\mathrm{p}=0.03)$, but the difference between these 2 modalities was not significant during flexion-extension ( $p=0.113)$ or lateral bending $(p=0.066)$. The $\mathrm{LZ}$ was significantly greater with bilateral UC than with bilateral pedicle screws during lateral bending $(\mathrm{p}=0.037)$ and axial rotation $(p=0.031)$, but not during flexion-extension ( $p$ $=0.113$ ). Bilateral UC resulted in a similar $\mathrm{LZ}$ as hybrid fixation in all directions of loading ( $p>0.1)$. The LZ with

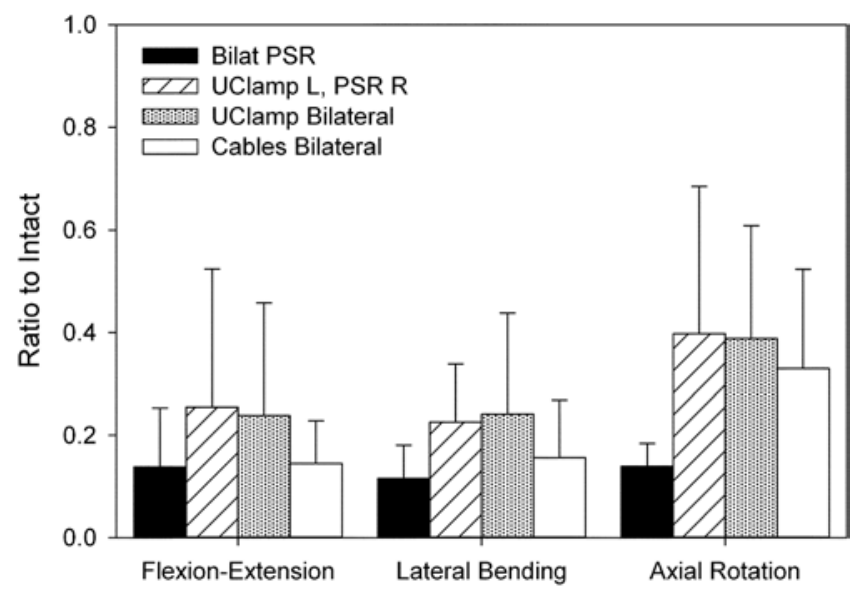

FIG. 3. Mean normalized bidirectional angular LZ across T4-10. The error bars show the standard deviation. 
bilateral cables was not significantly different with bilateral pedicle screws, hybrid fixation, or bilateral UC in any direction of loading $(\mathrm{p}>0.1)$.

The SZ (Fig. 4) for the bilateral pedicle screw condition was significantly smaller than with hybrid fixation during all directions of loading ( $p<0.02)$, except extension ( $p$ $=0.113$ ). Bilateral UC resulted in significantly greater SZ than bilateral pedicle screws during left and right lateral bending $(\mathrm{p}<0.004)$ and right axial rotation $(\mathrm{p}=0.048)$, but not during flexion $(p=0.056)$, extension ( $p=0.113)$, or left axial rotation $(\mathrm{p}=0.073)$. There was no difference in SZ between bilateral and hybrid UC fixation during any direction of loading $(p>0.1)$. Bilateral cables resulted in significantly greater SZ than bilateral pedicle screws during right and left lateral bending $(\mathrm{p}<0.04)$ and right axial rotation $(\mathrm{p}=0.048)$, but not during flexion $(\mathrm{p}=0.184)$, extension $(\mathrm{p}=0.113)$, or left axial rotation $(\mathrm{p}=0.079)$. There were no differences in SZ between bilateral cables, hybrid fixation, or bilateral UC in any direction of loading ( $\mathrm{p}>$ $0.1)$.

Analysis of segmental ROM, LZ, and SZ showed that there was a trend for decreased mobility of the segments toward the center of the instrumented construct (i.e., T68) compared with segments at the inferior and superior ends of the construct (T4-6 and T8-10; Fig. 5). This trend was apparent for all 4 constructs, especially during flexion-extension and lateral bending.

The relative contribution for ROM for T6-8 (the center of the construct) compared with T4-10 (the entire instrumented region) was not significantly different between any pair of constructs during flexion ( $p>0.08$; Fig. 5), extension ( $\mathrm{p}>0.15$; Fig. 5$)$, or right axial rotation ( $\mathrm{p}>0.4$; Fig. 5). However, during left lateral bending, the relative contribution of the most central segments (T6-T8) to the ROM compared with that of the entire instrumented section (T4-10) was significantly greater with bilateral UC than with bilateral pedicle screws $(p=0.023$; Fig. 5). The differences in the relative segmental ROM contribution between the other pairs of constructs were not significant ( $p>0.07)$. During right lateral bending, the contribution

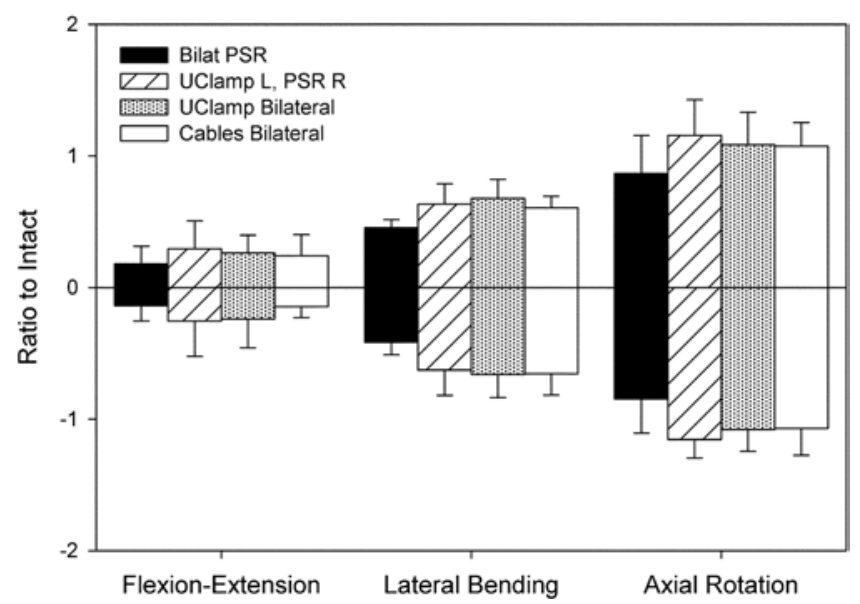

FIG. 4. Mean normalized unidirectional angular SZ across T4-10. Flexion, right lateral bending, and right axial rotation are represented as positive; extension, left lateral bending, and left axial rotation are represented as negative. The error bars show the standard deviation. of the most central segments (T6-8) to the ROM relative to that of T4-10 was significantly greater with hybrid fixation $(\mathrm{p}=0.004)$, bilateral UC $(\mathrm{p}=0.005)$, and bilateral cables $(\mathrm{p}=0.005)$ than with bilateral pedicle screws (Fig. 5). There were no significant differences between hybrid fixation, bilateral UC, and bilateral cables ( $p>0.9)$. During left axial rotation, the contribution to the ROM of the centrally instrumented segments (T6-8) relative to all instrumented sections (T4-10) was significantly greater with bilateral cables than with bilateral pedicle screws $(\mathrm{p}=0.021)$ and hybrid fixation $(p=0.025)$. There were no significant differences in the relative contribution to mobility among the remaining constructs during left axial rotation ( $p>0.06$ ), and there were no significant differences among any of the constructs during right axial rotation $(p>0.9)$.

There was a trend for the segmental LZ to decrease toward the centrally instrumented levels versus the superior and inferior ends of the instrumented constructs during flexion-extension and lateral bending, but not during axial rotation. There were no differences in the ratio of $\mathrm{LZ}$ at T6-8 versus T4-10 among the constructs during flexionextension ( $p=0.443)$, lateral bending $(p=0.360)$, or axial rotation $(\mathrm{p}=0.140)$.

The trend for decreased segmental SZ toward the centrally instrumented levels was similar to ROM in all directions of loading. There were no significant differences in the ratio of SZ at T6-8 to SZ at T4-10 among the constructs during flexion $(p=0.085)$, extension $(p=0.181)$, or right axial rotation $(\mathrm{p}=0.488)$. During left lateral bending, bilateral pedicle screws resulted in a smaller ratio than bilateral UC $(p=0.023)$ or bilateral cables $(p=0.044)$. During right lateral bending, the ratio of SZ at T6-8 to SZ at T4-10 with bilateral pedicle screws was significantly smaller than with hybrid fixation, bilateral UC, or bilateral cables $(p=0.002)$. During left axial rotation, the ratio of SZ at T6-8 to SZ at T4-10 with bilateral pedicle screws was significantly smaller than with bilateral cables $(\mathrm{p}=$ $0.017)$.

\section{Discussion}

The correction of scoliosis has been a common surgery since Harrington introduced rod instrumentation in the $1960 \mathrm{~s}^{7,11}$ The options for the treatment of scoliosis surgery have changed, with various shapes of cables, hooks, screws, and rods being used. This study compares the use of pedicle screws, sublaminar cables, and UC for instrumentation in the thoracic spine. Luque's sublaminar cable technique has been used since $1976^{15}$ with mixed clinical results and neurological complication rates ranging from $1 \%$ to $17 \% .^{21}$

The UC is a novel device that consists of 3 components: a woven polyester band, a titanium alloy clamp, and a locking screw. The UC is an alternative to cables for sublaminar fixation and has been used with good clinical results. In previous studies, using UC as a part of a hybrid construct was beneficial for achieving good correction in the coronal and axial planes and even better for correction in the sagittal plane.1,16,20 The rate of complications with UC was found to be lower than with cables (no complications reported by Viswanathan et al. or Lamartina et al.). ${ }^{14,20}$ The 

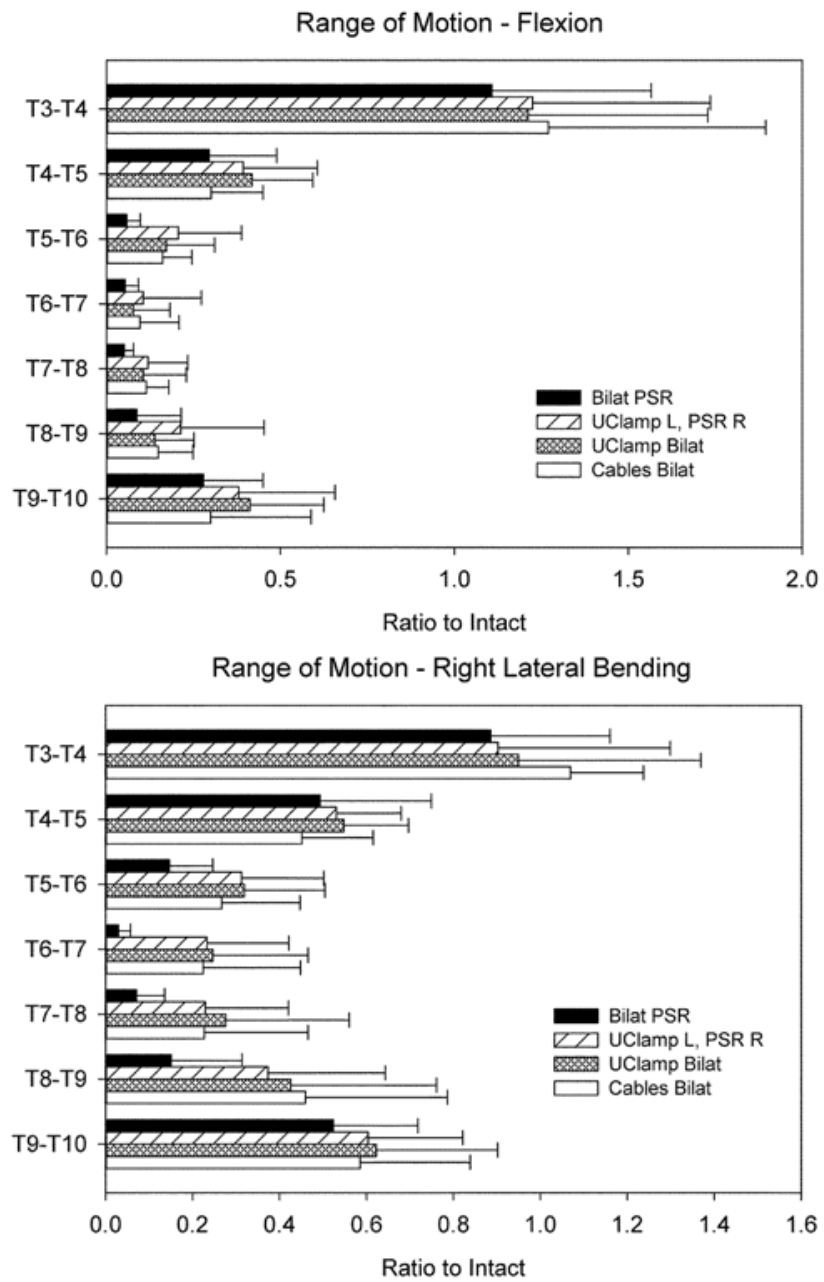

Range of Motion - Right Axial Rotation

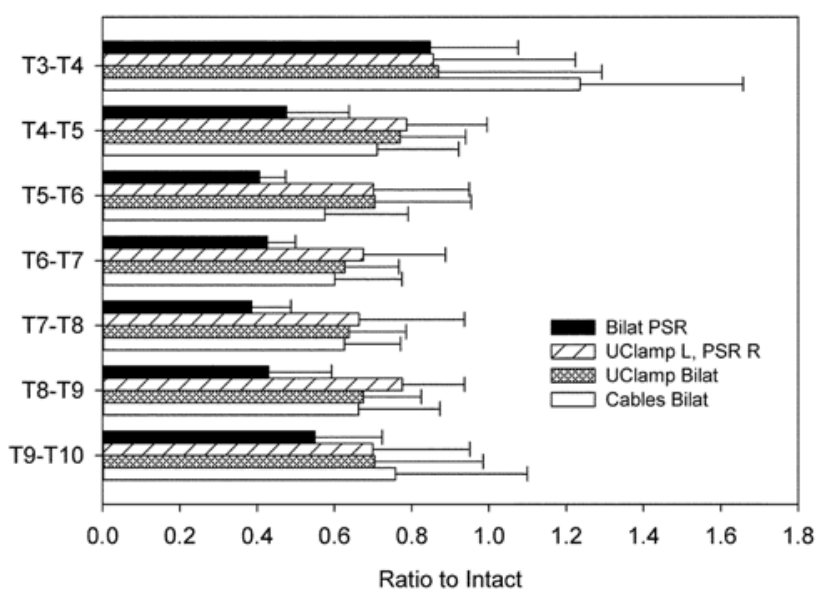

Range of Motion - Extension

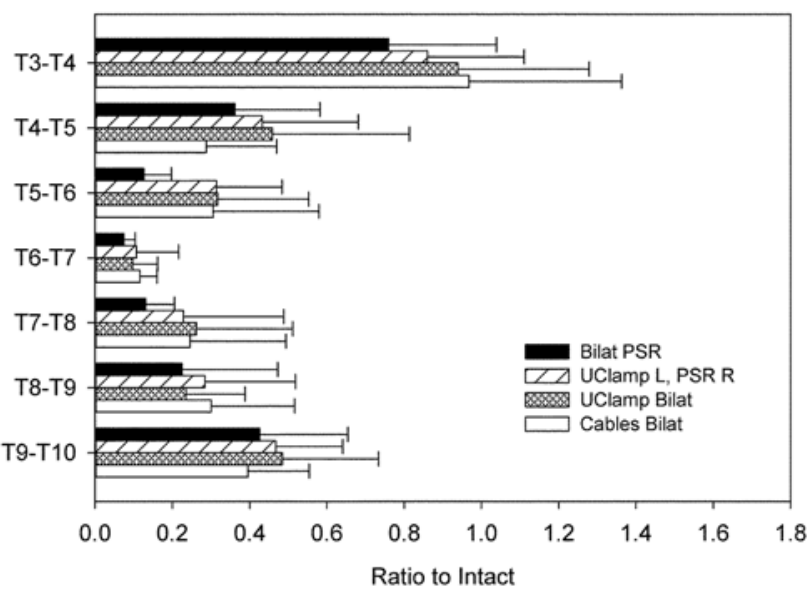

Range of Motion - Left Lateral Bending

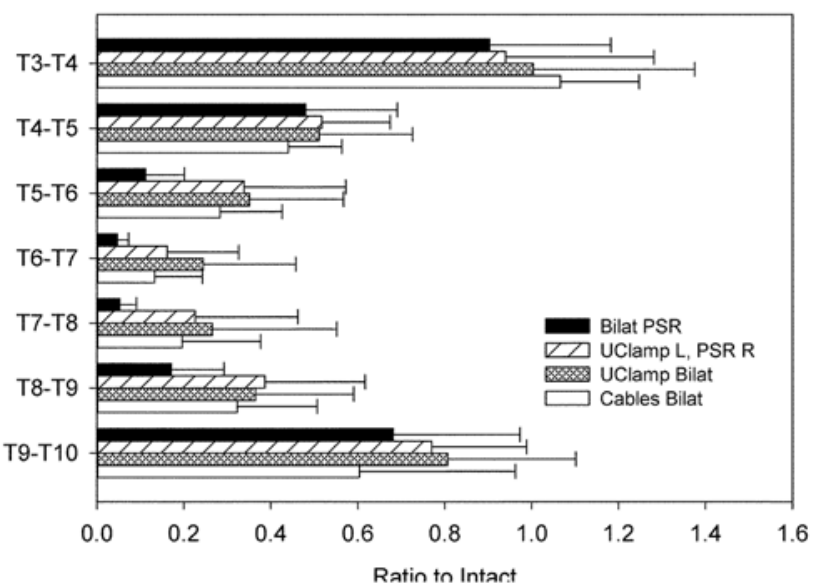

Range of Motion - Left Axial Rotation

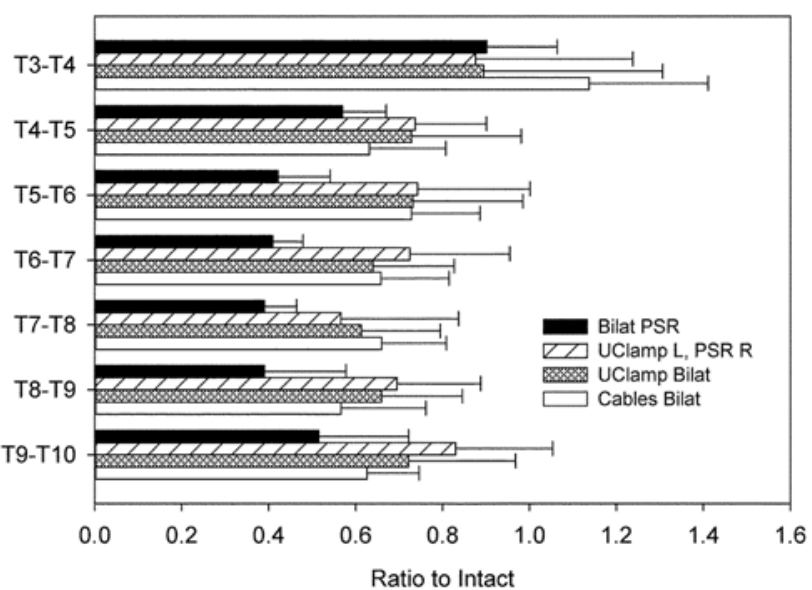

FIG. 5. Mean normalized unidirectional segmental angular ROM in each loading mode. The error bars show the standard deviation.

previous studies did not specifically address why a lower rate of complications would be found since UC is also sublaminarly passed; however, the UC polyester band allows for increased surface contact area and conformance around the bone than with a metal cable, potentially resulting in fewer complications.
Until now, there have been no biomechanical studies reported in the literature that evaluate how the UC affects ROM. Hongo et al. ${ }^{8}$ studied the pullout forces and failure mechanisms of UC compared with pedicle screws, laminar hooks, and sublaminar wiring in the thoracic spine. It was shown that UC offers a better distribution of 
stress over a larger area of bone than sublaminar cables or hooks, reducing the risk of failure at the device-bone interface. ${ }^{8}$ Other clinical studies support the idea that the $\mathrm{UC}$ reduces the risk of device-bone failure: there were no modifications of the monitored potentials and no laminar or UC breakage with curve reductions during scoliosis surgery $^{16}$ compared with a rate of $39 \%$ for total surgical complications when using cables, as reported by Girardi et al. ${ }^{6}$

As expected, we found that the ROM using a bilateral screw construct was smaller in all directions of movement than with bilateral UC or bilateral cables (Fig. 2). It is remarkable that the bilateral UC demonstrated no statistically significant difference in ROM from bilateral cables since the UC band is more flexible than the metal sublaminar cable. Previous research showed that the UC restricts motion in all 3 planes more than a dynamic posterior device (Wallis, Zimmer Spine SAS), with the greatest difference between devices observed during flexion. ${ }^{9}$ Similarly, we found $\mathrm{UC}$ to be most effective (smallest ratio to intact ROM) in the flexion-extension plane.

It was hypothesized that, due to its flexibility, UC would allow a smaller LZ but a larger ROM than sublaminar cables or all-screw constructs. This behavior was not observed: the LZ was larger with UC than with either sublaminar cables or the all-screw construct (Fig. 3). It may be that differences in allowed LZ between sublaminar cables and UC would not show up until later in the postoperative period when cables would have undergone stretching, but the UC, having more elasticity than a cable, would be expected to loosen less. Further testing is needed to verify this hypothesis. However, it should be noted that although the LZ was larger under the UC condition, it was still significantly less than under the intact condition (23\%-39\% of intact), so it would not be considered unstable in any direction of loading.

When we compared the mean ROM of the hybrid construct, which consisted of unilateral UC and limited pedicle screws/rods on the opposite side, to a bilateral UC construct, there were no statistically significant differences (Fig. 2). The same trends were observed with the LZ and SZ (Figs. 3 and 4). These results support the idea that, in a 7-vertebra construct, the UC used at the 5 internal vertebrae with pedicle screws at the distal ends imparts stability and stiffness across this long construct that are roughly equivalent to that of a long 3-point pedicle screwrod construct. Therefore, transitional segments or those at the vertex of a curve-where UC placement may be easier and safer than pedicle screw placement into a rotated and possibly malformed pedicle-should be a good alternative, as clinically suggested. ${ }^{14,18}$

We also studied the segmental motion of each construct and found that the bilateral UC construct allowed a greater proportion of the overall motion to occur near the center of the construct than the all-pedicle screw construct due to the lower stiffness of the UC (Fig. 5). This finding indicates that a construct consisting of distal pedicle screws with intervening UC may result in the more even distribution of forces along the construct, potentially reducing the incidence of pullout or loosening of the distal screws. Further research into such benefits is needed, potentially in- cluding instrumented pedicle screws for monitoring screw loads or computer simulations to study load distributions.

\section{Conclusions}

A 6-level (7-vertebra) construct utilizing the UC at all levels, except at the distal ends, provides flexibility intermediate to that of the intact spine and spine instrumented with an all-screw construct.

Our results support the use of UC as a therapeutic option for spinal stabilization because UC allows comparable stability to sublaminar cables. Similar stability is achieved between a hybrid construct that combines 3-point pedicle screw/rod fixation on one side and UC on the other side, bilateral UC, and bilateral sublaminar cables. Therefore, in apical levels, the use of bilateral UC provides equivalent stability to a 3-point pedicle screw/rod construct or sublaminar cable construct and avoids the clinical risks of pedicle screw placement or sublaminar cable placement in anatomically altered vertebrae.

\section{References}

1. Caekebeke P, Moke L, Moens P: Sublaminar devices for the correction of scoliosis: metal wire versus polyester tape. Acta Orthop Belg 79:216-221, 2013

2. Crawford NR, Brantley AG, Dickman CA, Koeneman EJ: An apparatus for applying pure nonconstraining moments to spine segments in vitro. Spine (Phila Pa 1976) 20:20972100, 1995

3. Crawford NR, Dickman CA: Construction of local vertebral coordinate systems using a digitizing probe. Technical note. Spine (Phila Pa 1976) 22:559-563, 1997

4. Crawford NR, Peles JD, Dickman CA: The spinal lax zone and neutral zone: measurement techniques and parameter comparisons. J Spinal Disord 11:416-429, 1998

5. Crawford NR, Yamaguchi GT, Dickman CA: A new technique for determining 3-D joint angles: the tilt/twist method. Clin Biomech (Bristol, Avon) 14:153-165, 1999

6. Girardi FP, Boachie-Adjei O, Rawlins BA: Safety of sublaminar wires with Isola instrumentation for the treatment of idiopathic scoliosis. Spine (Phila Pa 1976) 25:691-695, 2000

7. Harrington PR: Treatment of scoliosis. Correction and internal fixation by spine instrumentation. J Bone Joint Surg Am 44-A:591-610, 1962

8. Hongo M, Ilharreborde B, Gay RE, Zhao C, Zhao KD, Berglund LJ, et al: Biomechanical evaluation of a new fixation device for the thoracic spine. Eur Spine J 18:1213-1219, 2009

9. Ilharreborde B, Shaw MN, Berglund LJ, Zhao KD, Gay RE, An KN: Biomechanical evaluation of posterior lumbar dynamic stabilization: an in vitro comparison between Universal Clamp and Wallis systems. Eur Spine J 20:289-296, 2011

10. Jouve JL, de Gauzy JS, Blondel B, Launay F, Accadbled F, Bollini G: Use of the Universal Clamp for deformity correction and as an adjunct to fusion: preliminary results in scoliosis. J Child Orthop 4:73-80, 2010

11. Kim YJ, Lenke LG, Kim J, Bridwell KH, Cho SK, Cheh G, et al: Comparative analysis of pedicle screw versus hybrid instrumentation in posterior spinal fusion of adolescent idiopathic scoliosis. Spine (Phila Pa 1976) 31:291-298, 2006

12. La Rosa G, Giglio G, Oggiano L: Surgical treatment of neurological scoliosis using hybrid construct (lumbar transpedicular screws plus thoracic sublaminar acrylic loops). Eur Spine J 20 (Suppl 1):S90-S94, 2011

13. La Rosa G, Giglio G, Oggiano L: The Universal Clamp hy- 
brid system: a safe technique to correct deformity and restore kyphosis in adolescent idiopathic scoliosis. Eur Spine J 22 (Suppl 6):S823-S828, 2013

14. Lamartina C, Cecchinato R: Selective thoracolumbar instrumentation with pedicle screws and sublaminar bands (universal clamps) in adolescent idiopathic scoliosis. Eur Spine J 20:2286-2287, 2011

15. Luque ER: Segmental spinal instrumentation for correction of scoliosis. Clin Orthop Relat Res (163):192-198, 1982

16. Mazda K, Ilharreborde B, Even J, Lefevre Y, Fitoussi F, Penneçot GF: Efficacy and safety of posteromedial translation for correction of thoracic curves in adolescent idiopathic scoliosis using a new connection to the spine: the Universal Clamp. Eur Spine J 18:158-169, 2009

17. Panjabi MM: Biomechanical evaluation of spinal fixation devices: I. A conceptual framework. Spine (Phila Pa 1976) 13:1129-1134, 1988

18. Sales de Gauzy J, Jouve JL, Ilharreborde B, Blondel B, Accadbled F, Mazda K: Use of the Universal Clamp in adolescent idiopathic scoliosis. Eur Spine J 23 (Suppl 4):S446S451, 2014

19. Tegos S, Charitidis C, Korovessis PG: Hybrid circumferential fixation for degenerative lumbosacral spine disease: posterior lumbar interbody fusion plus universal clamp rod-band instrumentation: a novel technique for lumbosacral fixation. Spine (Phila Pa 1976) 39:E441-E449, 2014

20. Viswanathan A, Johnson KK, Whitehead WE, Curry DJ, Luerssen TG, Jea A: Hybrid spinal constructs using sublaminar polyester bands in posterior instrumented fusions in children: a series of 5 cases. Neurosurgery 66:862-867, 2010

21. Wilber RG, Thompson GH, Shaffer JW, Brown RH, Nash CL
Jr: Postoperative neurological deficits in segmental spinal instrumentation. A study using spinal cord monitoring. J Bone Joint Surg Am 66:1178-1187, 1984

22. Winter RB, Anderson MB: Spinal arthrodesis for spinal deformity using posterior instrumentation and sublaminar wiring. A preliminary report of 100 consecutive cases. Int Orthop 9:239-245, 1985

\section{Disclosure}

Dr. Loughran is an employee of Zimmer Spine. Dr. Crawford received grant support for this work that was directly paid to his institution. Funding was provided by Zimmer Spine.

\section{Author Contributions}

Conception and design: Crawford, Safavi-Abbasi. Acquisition of data: Rodriguez-Martinez, Safavi-Abbasi, Perez-Orribo, Newcomb, Reyes. Analysis and interpretation of data: Crawford, Safavi-Abbasi, Newcomb, Loughran. Drafting the article: RodriguezMartinez. Critically revising the article: Crawford, Loughran. Reviewed submitted version of manuscript: Crawford, Loughran. Statistical analysis: Newcomb. Administrative/technical/material support: Crawford. Study supervision: Theodore.

\section{Correspondence}

Neil R. Crawford, c/o Neuroscience Publications, Barrow Neurological Institute, St. Joseph's Hospital and Medical Center, 350 W. Thomas Rd., Phoenix, AZ 85013.email: neuropub@ dignityhealth.org. 